\title{
On Buffer-Assisted Opportunistic Routing Relying on Linear Transmission Activation Probability Space Partitioning for Relay-Aided Networks
}

\author{
Chen Dong, Jing Zuo, Lie-Liang Yang, Yongkai Huo, Soon Xin Ng, and Lajos Hanzo \\ School of ECS, University of Southampton, SO17 1BJ, United Kingdom \\ Tel: 0044-(0)23-8059 3364, Email: \{cd2g09,jz08r,lly,yh3g09,sxn,lh\}@ecs.soton.ac.uk, http://www-mobile.ecs.soton.ac.uk
}

\begin{abstract}
In this paper buffer-aided Opportunistic Routing (OR) was designed with the aid of the novel concept of linear Transmission Activation Probability Space (TAPS) partitioning invoked for relayassisted networks, which combines the benefits of both OR [1] and of buffer-aided transmissions [2]. More specifically, a packet may be transmitted from the Source Node (SN) to the Destination Node (DN) either directly or indirectly via one of the $M$ Relay Nodes (RNs), depending on the instantaneous channel qualities. The above-mentioned linear multi-dimensional TAPS partitioning concept is proposed for partitioning the transmission space into $(2 M+1)$ transmission regions plus an outage region, while ensuring that the number of input packets is equal to the number of output packets at each RN's buffer. The benefit of having a buffer and tolerating the associated delay is that the best channel is activated for transmission based on our linear TAPS partitioning method.
\end{abstract}

Index Terms-Cooperative communication, opportunistic routing, buffer, energy consumption, energy dissipation, channel space.

\section{INTRODUCTION}

Employing a relay between the Source Node (SN) and the Destination Node (DN) is one of the most attractive methods that can be used for minimizing the Energy Dissipation (ED). The scheme, which assumes that a packet is transmitted from the SN to the DN via a Relay Node (RN) results in a range of advantages over conventional single-hop communications. These advantages may include an extended coverage area, an improved link performance, high-flexibility network planning, etc. [3-5]. Opportunistic Routing (OR) $[1,6-8]$ was shown to be capable of further enhancing the system's throughput in relay-aided wireless transmission. For example, Liu et al. [6] illustrated that OR substantially increases both the achievable transmission reliability and the throughput by exploiting the broadcast nature of the wireless medium, where all transmissions can be overheard by multiple neighbours. Then Biswas and Morris [1] proposed an 'Extremely' Opportunistic Routing (ExOR) scheme, which relied on the expected end-to-end transmission delay as the metric used for deciding on the priority order of selecting a RN from the potential forwarder set. The proposed routing regime intrinsically amalgamated the routing protocol and the Medium Access Control (MAC) protocol for the sake of increasing the attainable throughput of multi-hop wireless networks. Their solution [1] also exploited the less reliable long-distance links, which would have been ignored by traditional routing protocols. Zeng et al. [7] proposed multi-rate OR by incorporating rate-adaptation into their candidate-selection algorithm, which was shown to achieve a higher throughput and lower delay than the corresponding traditional single-rate routing and its opportunistic single-rate routing counterpart. Moreover, in our previous work [8], we proposed energy-efficient cross-layer aided OR

The financial support of the RC-UK under the auspices of the India-UK Advanced Technology Centre, the support of European Research Council's Advanced Fellow Grant, the support of the EU's Concerto project is gratefully acknowledged. for ad hoc networks and analytically formulated both the normalized energy consumption and the end-to-end throughput. The simulation results demonstrated that the proposed OR algorithm has a lower normalized energy consumption and higher end-to-end throughput than the traditional multi-hop routing algorithm. Additionally, our previous contributions $[2,9,10]$ proposed a buffer-aided transmission scheme, namely the Multihop Diversity (MHD) transmission philosophy, which relies on temporarily storing the received packets and on activating the specific channel having the highest instantaneous Signal-to-Noise Ratio (SNR). Both our simulation results and theoretical analysis demonstrated that MHD transmissions are capable of achieving a substantial selection diversity gain. Very recently, a relayrelation scheme was proposed in [11], while full-duplex relaying was discussed in [12]. As a further advance, adaptive link selection was proposed in [13].

A non-linear Transmission Activation Probability Space (TAPS) partitioning method was proposed in our previous paper [14], which combines the advantages of both buffer-aided transmissions and of OR. Although, it was conceived for a simple single-relay assisted network, yet it imposed a substantial complexity. Hence in this contribution a new low-complexity linear multi-dimensional TAPS partitioning method is proposed for the sake of guaranteeing the system's stability, namely, that the number of input packets equals the number of output packets at each RN's buffer. The proposed linear TAPS partitioning method is then investigated in Rayleigh fading channels. Explicitly, we derive a closed-form equation for the calculation of the activation probability of a specific hop. Furthermore, we develop close-form formulas for the average end-to-end energy dissipation and for the Outage Probability (OP). Note that the concepts of block delay and packet delay were defined in [2]. The block delay is inverse by proportional to the end-to-end throughput. In our scheme, the proposed linear multi-dimensional TAPS partitioning method becomes capable of reducing both the system's energy dissipation and its OP, whilst increasing its throughput. On the other hand, the packet delay represents the end-to-end delay for a specific packet. In our scheme, the packet delay is larger than that of the conventional scheme, but this is typically acceptable in a non-interactive delaytolerant system. The remainder of this paper is organized as follows. Section II presents our system model. Then Section III details the linear multi-dimensional TAPS partitioning philosophy and elaborates on our new channel activation scheme. In Section IV, we provide our numerical and simulation results. Finally, our conclusions are offered in Section V.

\section{SySTEM MODEL}

Our multiple-relay-aided network considered in this paper is shown in Fig. 1, which consists of a SN $S, M$ buffer-aided RNs $R_{m}, m=$ $1 \ldots M$, and a DN $D$. The distances between each corresponding pairs of nodes are $d_{S R_{m}}, d_{S D}$ and $d_{R_{m} D}$. We assume that no transmissions 


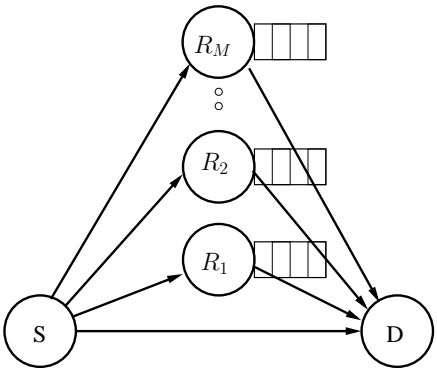

Fig. 1. System model for an $M$-relay-aided network, where each relay has a buffer. The SN $S$ sends messages to the DN $D$, either directly or indirectly, via $\mathrm{RN} R_{m}, m=1 \ldots M$.

take place between any of the RNs. The classic Decode-and-Forward (DF) protocol [15] is employed at each RN for relaying the signals.

Moreover, we assume that the signals are transmitted on the basis of Time Slots (TSs). Therefore, only a single channel is activated in each TS. Note that if no packets are stored in RN $R_{m}$, the $m$ th RNDN channel must not be activated. By contrast, if the buffer of RN $R_{m}$ is full, the $\mathrm{SN}-\mathrm{R}_{m}$ channel must not be activated. We assume furthermore that:

- The SN always has packets to send, which hence facilitates for the relay-aided network to operate in its steady state.

- Both the SN and DN are capable of storing an infinite number of packets. By contrast, the RN can only store a maximum of $B$ packets.

- The Rayleigh fading processes of the multiple channels are independent. The fading envelope of a given hop remains constant within a packet's duration, but it is independently faded from one packet to another.

- Each node accurately adjusts its transmission power between zero and the maximum transmit power $P_{\max }$ to achieve the required received SNR of $\gamma_{T h}$.

- Only a single packet is transmitted in each TS, when the corresponding link is activated.

- Our relay-aided network protocol provides every node with the global Channel Quality (CQ) knowledge and Buffer Fullness (BF) knowledge of the RNs within a given TS. All the operations of the proposed protocol are assumed to have been carried out without a delay and without errors.

Furthermore, the pathloss is assumed to obey the negative exponential law of $d^{-\alpha}$, where $\alpha$ is the pathloss exponent, having a value between 2 to 6 . It is also assumed that the instantaneous CQ of TS $t$ between each node pairs is denoted by $\gamma_{S R_{m}}=\bar{\gamma}_{S R_{m}} \gamma_{S R_{m}}^{f a d}$, $\gamma_{S D}=\bar{\gamma}_{S D} \gamma_{S D}^{f a d}$ and $\gamma_{R_{m} D}=\bar{\gamma}_{R_{m} D} \gamma_{R_{m} D}^{f a d}$, where $m=1 \ldots M$, the values marked by the upper bar, as in $\bar{\gamma}$ represent the average receive SNR, while the values marked by the superscript 'fad' as in $\gamma^{\text {fad }}$ are the instantaneous fading values. The instantaneous transmit power $\mathcal{E}_{S D}, \mathcal{E}_{S R_{m}}$ or $\mathcal{E}_{R_{m} D}$ of each node can then be calculated with the aid of $\kappa=9.895 \times 10^{-05}$ and the noise power of $N=10^{-14} \mathrm{~W}$, which corresponds to a receiver sensitivity of $-110 \mathrm{dBm}$. An example of calculating $\mathcal{E}_{S D}$ is given by

$$
\mathcal{E}_{S D}=\frac{\gamma_{T h}}{\gamma_{S D}^{\text {fad }}} \frac{d_{S D}^{\alpha} N}{\kappa}, \mathcal{E}_{S D} \leq P_{\max } .
$$

\section{TheORETICAL ANALYSIS}

In this section, the energy dissipation, OP and average block delay bound will be analyzed. Based on Fig. 1, we can now infer that if every RN has a buffer size of $B$ packets, the following events may occur. 1 ) Firstly, the buffer of a RN may be empty at a certain instant $t$. In this case, this RN cannot be the transmit node, since it has no data to transmit. 2 ) Secondly, the buffer of a RN may be full at a certain instant $t$. Hence this RN cannot act as the receive node, since it cannot accept further packets. Therefore, based on the same assumption as in [2], our lower-bound expressions of the energy dissipation and outage probability are derived by relaxing the above-mentioned constraints. Instead, we assume that each RN has an unlimited buffer size and that a node always has packets to transmit.

For this specific system, our main assumption is that the number of packets conveyed from SN to RN should be the same as those transmitted from RN to DN, which implies that the SN-RN channel and RN-DN channel should have the same probability of being activated. This assumption is automatically satisfied for $d_{S R_{m}}=$ $d_{R_{m} D}$, since we have identical channel conditions in both hops. However, for the sake of dealing with the specific conditions, when we have $d_{S R_{m}} \neq d_{R_{m} D}$, the discussion of channel-activation may be commenced from the concept of TAPS.

\section{A. Multi-dimensional Transmission Activation probability space}

The concept of three-dimensional CPS was originally proposed in our previous paper $[14,16]$. The CPS is a Cartesian coordinate system-based space. Each coordination represents the instantaneous fading value of a channel. In this contribution, the $(2 M+1)$ channels form a $(2 M+1)$-dimensional TAPS. For example, given a TS, the instantaneous fading values $\left(\gamma_{S D}^{f a d}, \gamma_{S R_{1}}^{f a d}, \cdots, \gamma_{S R_{M}}^{f a d}, \gamma_{R_{1} D}^{f a d}, \cdots, \gamma_{R_{M} D}^{f a d}\right)$ of all the channels is mapped to the point $\left(\gamma_{S D}^{f a d}, \gamma_{S R_{1}}^{f a d}, \cdots, \gamma_{S R_{M}}^{f a d}, \gamma_{R_{1} D}^{f a d}, \cdots, \gamma_{R_{M} D}^{f a d}\right)$ in this $(2 M+1)$-dimensional space. In the following, let us use the subscript $1,2, \cdots,(2 M+1)$ to represent the channels $S D, S R_{1}, \cdots, S R_{M}, R_{1} D, \cdots, R_{M} D$.

Based on our assumptions, a channel will be activated, when at least one of the $(2 M+1)$ channels is available. By contrast, when all the CQs are lower than their corresponding threshold, an outage occurs. The resultant outage probability bound $P_{\text {out }}$ is formulated as:

$$
P_{\text {out }}=\prod_{i=1}^{2 M+1}\left(1-e^{-\frac{\gamma_{T h} d_{i}^{\alpha} N}{P_{\max } \kappa}}\right) .
$$

B. Linear TAPS Division Relying on an Infinite Maximum Transmission Power

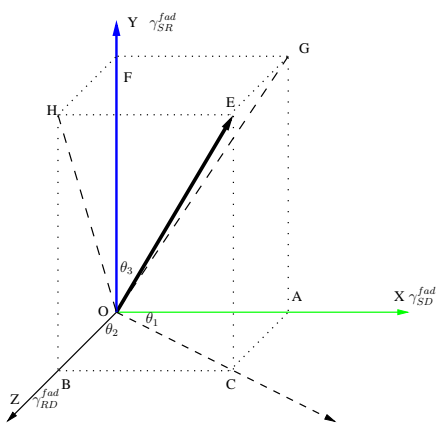

Fig. 2. The transmission activation probability space (TAPS) is partitioned by a line $\mathrm{OE}$. The angles between $\mathrm{OE}$ and each coordinate are given by $\left(\theta_{1}, \theta_{2}, \cdots, \theta_{2 M+1}\right)$. The TAPS can be partitioned into the $(2 M+1)$ regions. This figure is an example of the TAPS for $M=1$, where the axis $X, Y$ and $Z$ represent the instantaneous fading values of SN-DN, SN-RN and RN-DN channels, given by $\gamma_{S D}^{\text {fad }}, \gamma_{S R}^{\text {fad }}$ and $\gamma_{R D}^{\text {fad }}$, respectively.

In order to elaborate on the principle of linear TAPS, let us assume that the maximum transmission power is infinity. Hence, the OP always becomes negligible. We also assume that the line $\mathrm{OE}$ in the TAPS of Fig. 2 emerges from the origin and has an angle of $\left(\theta_{1}, \theta_{2}, \cdots, \theta_{2 M+1}\right)$ with respect to each of the coordinate axes. For a specific TS, the instantaneous fading values 
$\left(\gamma_{1}^{\prime f a d}, \gamma_{2}^{\prime f a d}, \cdots, \gamma_{2 M+1}^{\prime f a d}\right)$ are mapped to the point E' in TAPS, where the angles of OE' are $\left(\theta_{1}^{\prime}, \theta_{2}^{\prime}, \cdots, \theta_{2 M+1}^{\prime}\right)$. The channel $i$ will be activated when we have 1) $\theta_{i}^{\prime}<\theta_{i}$ and 2) $\theta_{j}^{\prime} \geq \theta_{j}$, $j=1,2, \cdots, 2 M+1$ and $j \neq i$, which physically means that $\mathrm{E}^{\prime}$ is in the region of the $i$ th channel in the TAPS. This hop-selection criterion will be used in our simulations. For example in Fig. 2, if $E^{\prime}$ is in the region of OACEG, OBHEC, OFGEH or in the corresponding extension regions, the $\mathrm{SD}$, RD or $\mathrm{SR}$ channel will be activated, respectively.

The specific angles between $\mathrm{OE}$ and each coordinate axis may then be found by a carefully designed algorithm. Again, this angle will be used for deciding upon which particular hop will be activated, because it determines which region the point E' belongs to. Let us proceed by describing the line $\mathrm{OE}$ with the aid of its Cartesian coordinates. As seen in Fig. 2, we have $O B=O A \cdot \arctan \left(\theta_{2}\right), O F=C E=$ $O C \cdot \arctan \left(\theta_{3}\right)=O A \cdot \csc \left(\theta_{2}\right) \cdot \arctan \left(\theta_{3}\right)$ and $\theta_{1}=\frac{\Pi}{2}-\theta_{2}$. Based on the same process, it is straightforward to describe the line OE,

$$
\begin{aligned}
\gamma_{S D}^{f a d} & =\gamma_{S D}^{f a d} \cdot \arctan \left(\theta_{2}\right)=\gamma_{S D}^{f a d} \cdot \csc \left(\theta_{2}\right) \cdot \arctan \left(\theta_{3}\right)=\cdots \\
& =\gamma_{S D}^{f a d}\left[\prod_{i=2}^{2 M} \csc \left(\theta_{i}\right)\right] \cdot \arctan \left(\theta_{2 M+1}\right)
\end{aligned}
$$

which can be written compactly as $\gamma_{S D}^{f a d} \beta_{1}=\gamma_{S D}^{f a d} \beta_{2}=\cdots=$ $\gamma_{S D}^{f a d} \beta_{2 M+1}$, with $\beta_{1}=1$. Let us now consider the SD hop as an example for the calculation of $P_{S D}$ and $E_{S D}$. The fading value of the SD channel may be in the range of $(0, \infty)$. If the fading value of the SD channel is given by $\gamma_{S D}^{\prime \text { fad }}$, the fading value of the $i$ th channel cannot exceed $\beta_{i} \gamma_{S D}^{\prime f a d}$, otherwise the $i$ th channel would be activated. Based on this principle and assuming that we have $T h_{u p}=\infty$ and $T h_{\text {low }}=0$, the activation probability $P_{S D}$ of the SD hop may be calculated as

$$
P_{S D}=\int_{T h_{l o w}}^{T h_{u p}} e^{-\beta_{1} \gamma_{S D}^{f a d}} \prod_{j=2}^{2 M+1}\left[\int_{0}^{\beta_{j} \gamma_{S D}^{f a d}} e^{-\gamma_{j}^{f a d}} d \gamma_{j}^{f a d}\right] d \gamma_{S D}^{f a d}
$$

After further manipulation, we arrive at:

$$
P_{S D}=\sum_{K=0}^{2 M+1}(-1)^{K}[\underbrace{\sum_{i_{1}=2}^{2 M+1} \cdots \sum_{i_{K}=2}^{2 M+1}}_{\substack{i_{l} \neq i_{h} \\ l, h=1,2, \cdots, K}} \frac{-\left.e^{-\beta_{1} \gamma_{S D}^{f a d} \sum_{j=2}^{K} \beta_{j}}\right|_{T h_{\text {low }}} ^{T h_{\text {up }}}}{\sum_{j=2}^{K} \beta_{j}}] .
$$

Correspondingly, the energy dissipation $E_{S D}$ of the SD hop may be formulated as:

$$
\begin{aligned}
& E_{S D} \\
& =\int_{T h_{\text {low }}}^{T h_{u p}} \frac{\gamma_{T h} d_{S D}^{\alpha} N}{\beta_{1} \gamma_{S D}^{f a d} \kappa} e^{-\beta_{1} \gamma_{S D}^{f a d}} \prod_{j=2}^{2 M+1}\left[\int_{0}^{\beta_{j} \gamma_{S}^{f a d} D} e^{-\gamma_{j}^{f a d}} d \gamma_{j}^{f a d}\right] d \gamma_{S D}^{f a d} \\
& =\frac{\gamma_{T h} d_{S D}^{\alpha} N}{\beta_{1} \kappa} \sum_{K=0}^{2 M+1}(-1)^{K} \\
& \times[\underbrace{\sum_{i_{1}=2}^{2 M+1} \cdots \sum_{i_{K}=2}^{2 M+1}}_{\substack{i_{l} \neq i_{h} \\
l, h=1,2, \cdots, K}}\left(E i\left(-\frac{T h_{\text {low }}}{\beta_{1} \sum_{j=2}^{K} \beta_{j}}\right)-E i\left(-\frac{T h_{\text {up }}}{\beta_{1} \sum_{j=2}^{K} \beta_{j}}\right)\right)
\end{aligned}
$$

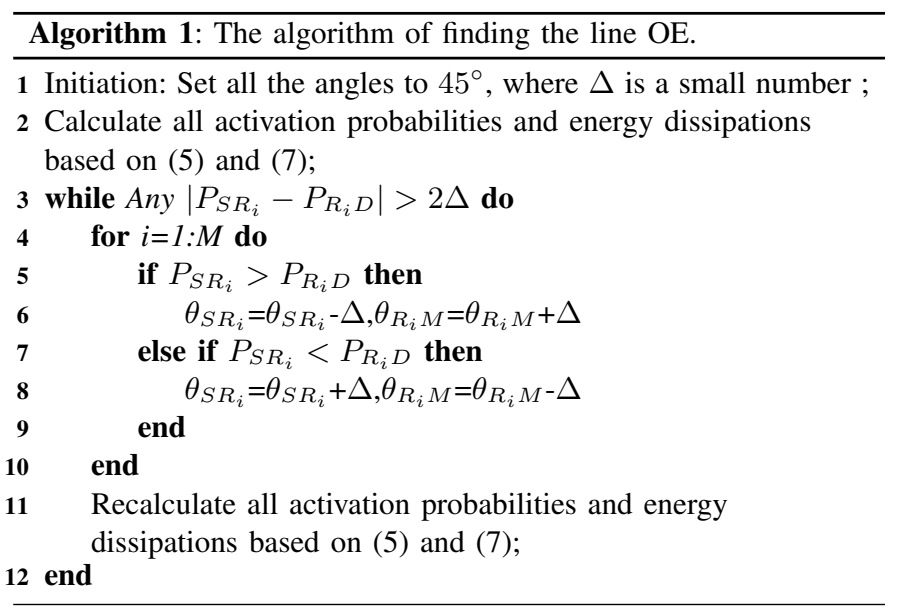

where $E i$ is defined in [17](8.211.1). Similarly to the SD hop, the activation probability and energy dissipation of all other hops can be calculated using a similar procedure. However, the channel activation probability $P_{S R_{i}}$ may not be the same as $P_{R_{i} D}$, because the distance $d_{S R_{i}}$ and $d_{R_{i} D}$ may be different. Therefore, below an algorithm is proposed for guaranteeing that the constraint of $P_{S R_{i}}=P_{R_{i} D}$ is satisfied by appropriately adjusting the angles of $\theta_{S R_{i}}$ and $\theta_{R_{i} D}$, which corresponds to adjusting the link activation probability, by allocating the appropriate activation region in TAPS. For example, if we have $P_{S R_{i}}<P_{R_{i} D}$, the corresponding angle of the $S R_{i}$ channel will be increased in order to increase the probability of this hop being activated. Naturally, at the same time $\theta_{R_{i} D}$ has be reduced based on the same principle.

\section{Linear TAPS Division for a Finite Maximum Transmission Power}

In Section III-B, the outage region of the TAPS was temporarily ignored, since the maximum transmission power was assumed to be $P_{\max }=\infty$. In this section, let us assume that $P_{\max }$ has a finite value. Thus, the outage threshold associated with each dimension of Fig. 2 will affect the results. The calculations of both the channel activation probability and of the energy dissipation are similar to those of (5) and (7). However, both the upper and lower limits of the integrals become different.

Let us define the outage threshold for the $i$ th dimension of the TAPS in the form of it SNR as $\gamma_{i}^{\text {out }}=-\frac{\gamma_{T h} d_{i}^{\alpha} N}{P_{\max } \kappa}$. There exists a point $G_{i}$ along the line $\mathrm{OE}$, where the projection of $G_{i}$ on the $i$ th dimension becomes $\gamma_{i}^{\text {out }}$. The coordinates of $G_{i}$ are

$$
G_{i}=\left(\frac{\gamma_{i}^{\text {out }} \beta_{1}}{\beta_{i}}, \frac{\gamma_{i}^{\text {out }} \beta_{2}}{\beta_{i}}, \cdots, \frac{\gamma_{i}^{\text {out }} \beta_{2 M+1}}{\beta_{i}}\right) .
$$

Having obtained the coordinates of all $G_{i}, i=1,2, \cdots,(2 M+1)$, we sort the length of all the lines $O G_{i}$, representing the thresholds in OE, yielding, $O G_{h_{1}} \geq O G_{h_{2}} \geq \cdots \geq O G_{h_{2 M+1}}$. This sorting operation will create a list of the number of available channels in various ranges of $\mathrm{OE}$. Let us elaborate a little further by considering an example of the calculation of the selection probability for the SD hop with the aid of (5). The projections of all the different thresholds $G_{h_{i}}$ onto the SD axis of Fig. 2 are $\frac{\gamma_{h_{1}}^{\text {out }} \beta_{1}}{\beta_{h_{1}}} \geq \frac{\gamma_{h_{2}}^{\text {out }} \beta_{1}}{\beta_{h_{2}}} \geq \cdots \geq \frac{\gamma_{h_{2 M+1}}^{\text {out }} \beta_{1}}{\beta_{h_{2 M+1}}}$ Therefore, when we have $\gamma_{S D} \in\left[\frac{\gamma_{h_{1}}^{\text {out }} \beta_{1}}{\beta_{h_{1}}}, \infty\right)$, all the channels are , available to be activated, while for $\gamma_{S D} \in\left[\frac{\gamma_{h_{n+1}}^{\text {out }} \beta_{1}}{\beta_{h_{n+1}}}, \frac{\gamma_{h_{n}}^{\text {out }} \beta_{1}}{\beta_{h_{n}}}\right)$, any channel associated with a channel index of $n^{\prime} \leq n$ is located in the outage region. Hence, these channels cannot be activated. Thus, the closed-form expression of the activation probability of the SD hop 
becomes

$$
\begin{aligned}
& P_{S D}=\sum_{n=1}^{2 M+1} \prod_{j=1}^{n-1}\left(1-e^{-\frac{\gamma_{h_{j}}^{\text {out }} \beta_{1}}{\beta_{h_{j}}}}\right) \int_{\max \left(\gamma_{1}^{\text {out }}, T h_{S D_{n-1}}\right)}^{T h_{S D_{n}}} e^{-\beta_{1} \gamma_{S D}^{f a d}} \\
& \times \prod_{\substack{j=2 \\
j \neq h_{n^{\prime}-1} \\
n^{\prime} \leq n}}^{2 M+1}\left[\int_{0}^{\beta_{j} \gamma_{S D}^{f a d}} e^{-\gamma_{j}^{f a d}} d \gamma_{j}^{f a d}\right] d \gamma_{S D}^{f a d} \\
& =\sum_{n=1}^{2 M+1} \prod_{j=1}^{n-1}\left(1-e^{-\frac{\gamma_{h_{j}}^{\text {out }} \beta_{1}}{\beta_{h_{j}}}}\right) \sum_{K=0}^{2 M+1}(-1)^{K} \underbrace{\sum_{i_{1}=2}^{2 M+1} \cdots \sum_{i_{K}=2}^{2 M+1}}_{i_{l} \neq h_{n^{\prime}-1}} \\
& n^{\prime} \leq n \\
& \begin{array}{l}
n^{\prime} \leq n \\
i_{l} \neq i_{h}
\end{array} \\
& l, h=1,2, \cdots, K \\
& \times \frac{-\left.e^{-\beta_{1} \gamma_{S D}^{f a d} \sum_{j=2}^{K} \beta_{j}}\right|_{\max \left(\gamma_{1}^{\text {out }}, T h_{S D_{n-1}}\right)} ^{T h_{S D_{n}}}}{\sum_{j=2}^{K} \beta_{j}},
\end{aligned}
$$

where we have $T h_{S D_{n}}=\frac{\gamma_{h_{n}}^{\text {out }} \beta_{1}}{\beta_{h_{n}}}$ and upon setting $T h_{S D_{0}}=\infty$, $h_{0}=0$. Correspondingly, the energy dissipation $E_{D}$ of SD the hop is given by (11). Similarly, the activation probability and the energy dissipation related to all other channels may be calculated, leading to similar closed-form expressions. The TAPS-based Algorithm 1 should be updated for the finite maximum transmission power by substituting (5) and (7) with (10) and (11).

Finally, let us now formulate the end-to-end energy dissipation bound $\overline{\mathcal{E}}_{S D}$, which is the ratio of the total energy dissipation to the end-to-end packet throughput, yielding

$$
\overline{\mathcal{E}}_{S D}=\frac{E_{S D}+\sum_{i=1}^{M}\left(E_{S R_{m}}+E_{R_{m} D}\right)}{P_{S D}+\sum_{i=1}^{M}\left(P_{S R_{m}}+P_{R_{m} D}\right)} .
$$

The end-to-end throughput $\Phi_{S D}$ and the end-to-end block delay $D_{S D}$ are

$$
\begin{gathered}
\Phi_{S D}=P_{S D}+\sum_{i=1}^{M}\left(P_{S R_{m}}+P_{R_{m} D}\right) / 2 . \\
D_{S D}=1 / \Phi_{S D} .
\end{gathered}
$$

Note that all the analysis is based on assuming an infinite buffer size. In the next section we show that the simulation results approach the analytical bound upon increasing buffer size.

\section{Performance Results}

In this section, we provide a range of numerical and/or simulation results for characterizing both the energy dissipation and the OP of the relay-aided network considered in order to illustrate the effects of both the position of the RNs and of the RNs' buffer size $B$. In all experiments, the $\mathrm{SN}$ is at the position $[100 \mathrm{~m}, 100 \mathrm{~m}]$, while DN is at the position $[900 \mathrm{~m}, 100 \mathrm{~m}]$. The parameters of $N$ and $\kappa$ are $N=10^{-14}$ and $\kappa=9.895 \times 10^{-05}$. For convenience, "normalized energy dissipation" refers to the "Average normalized end-to-end energy dissipation per packet". The label 'OR+buffer+linear+bound' indicates the values calculated from (2) and (12) when employing the proposed scheme, which combines the benefits of both OR and buffer-aided transmission associated with linear multi-dimensional TAPS partitioning scheme. By contrast, the simulation based values are represented by the label 'OR+buffer+linear+sim'. The buffer size ranges from $B=1$ to $B=256$ packets. Additionally, 'OR simulation' represents the simulation based values, when only the OR is employed [1].
First, we consider a 2-relay-aided network as our introductory example, where $M=2$. The label of ' $\mathrm{T} 1$ ' ' is used to indicate that $R_{1}$ is at the position $(500 \mathrm{~m}, 500 \mathrm{~m})$ and $R_{2}$ is at the position $(400 \mathrm{~m}, 300 \mathrm{~m})$, while 'T2' represents that $R_{1}$ is at the position $(366 \mathrm{~m}, 100 \mathrm{~m})$ and $R_{2}$ is at the position $(633 \mathrm{~m}, 100 \mathrm{~m})$.

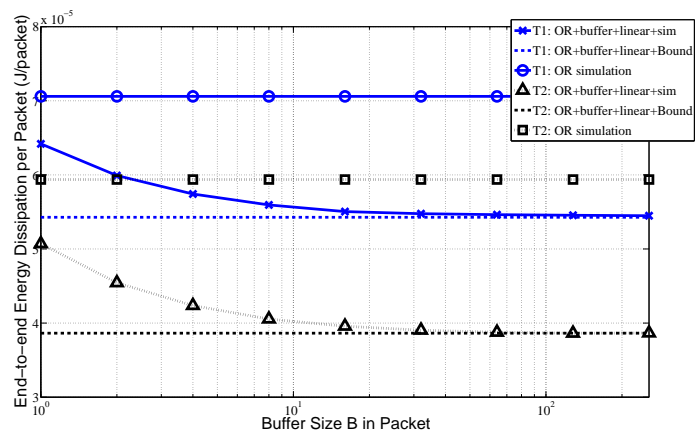

Fig. 3. Average normalized end-to-end energy dissipation ( $\mathrm{J} /$ packet) as defined in (12) when the pathloss factor is $\alpha=2$. The theoretical curve was evaluated from (10).

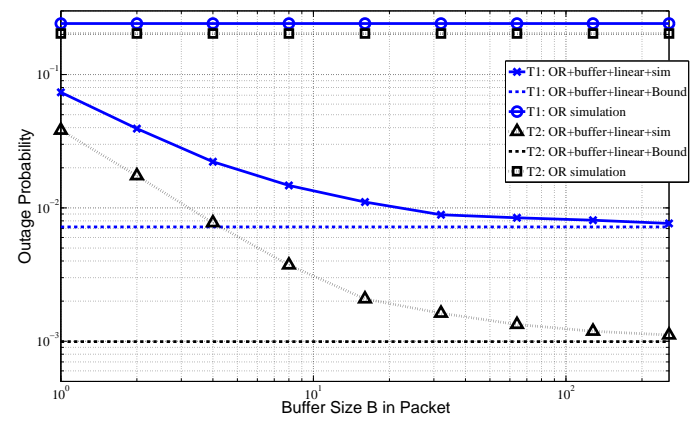

Fig. 4. The simulated and theoretical outage probability evaluated from (11)

Fig. 3 and Fig. 4 characterize the impact of both the RNs' buffer size and the RNs' position on the normalized end-to-end energy dissipation and the outage probability, respectively. Observe in both Fig. 3 and Fig. 4 that the simulation based performance curves quantify, how the energy dissipation and the OP is reduced upon increasing the buffer-size. Furthermore, these curves gradually approach the theoretical energy dissipation bound and OP bound, respectively, as the buffer size $B$ increases. Moreover, the 'OR+buffer+linear' scheme achieves a lower normalized end-to-end energy dissipation and a lower OP than those of the 'OR' scheme, since the proposed bufferaided OR relying on our linear multi-dimensional TAPS partitioning succeeds in efficiently exploiting the instantaneous CQs for activating the most appropriate channel for each packet's transmission, whilst simultaneously ensuring that the number of input packets equals that of the output packets at each RN's buffer. Moreover, as evidenced by Fig. 3 the energy dissipation was reduced by $22.8 \%$ for ' $\mathrm{T} 1$ ' and by $34.9 \%$ for ' $\mathrm{T} 2$ '.

Let us now investigate the effects of the number of RNs on the end-to-end energy dissipation, for $M=1,2,3$. For $M=1, R_{1}$ is at the position of $[400 \mathrm{~m}, 300 \mathrm{~m}]$, while for $M=2, R_{1}$ is at the position of $[400 \mathrm{~m}, 300 \mathrm{~m}]$ and $R_{2}$ is at the position of $[500 \mathrm{~m}, 500 \mathrm{~m}]$. Finally, for $M=3, R_{1}$ and $R_{2}$ are at the same position as those of $M=2$ but we have additional $\mathrm{RN} R_{3}$, which is at the position of $[700 \mathrm{~m}, 200 \mathrm{~m}]$. Fig. 5 shows that the scenario associated with the label ' $\mathrm{M}=3$ ' has the lowest end-to-end energy dissipation for both the conventional OR scheme and for our proposed scheme. Again, the end-to-end energy dissipation decreases for $M=1,2,3$ as the buffer size $B$ increases. When the buffer-size $B$ is sufficiently large, 


$$
\begin{aligned}
& E_{S D}=\sum_{n=1}^{2 M+1} \prod_{j=1}^{n-1}\left(1-e^{-\frac{\gamma_{h}^{\text {out }} \beta_{1}}{\beta_{h}}}\right) \int_{\max \left(\gamma_{1}^{\text {out }}, T h_{S D_{n-1}}\right)}^{T h_{S D_{n}}} \frac{\gamma_{T h}}{\beta_{1} \gamma_{S D}^{f a d}} \frac{d_{S D}^{\alpha} N}{\kappa} e^{-\beta_{1} \gamma_{S D}^{f a d}} \times \prod_{\substack{j=2 \\
j \neq h \\
n^{\prime}-1 \\
n^{\prime} \leq n}}^{2 M+1}\left[\int_{0}^{\beta_{j} \gamma_{S D}^{f a d}} e^{-\gamma_{j}^{f a d}} d \gamma_{j}^{f a d}\right] d \gamma_{S D}^{f a d}
\end{aligned}
$$

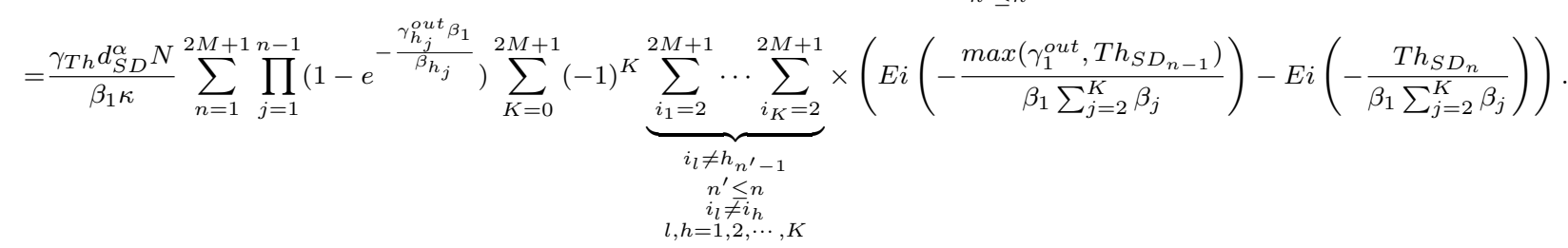

the simulation based performance of the proposed scheme indeed approaches its corresponding bound.

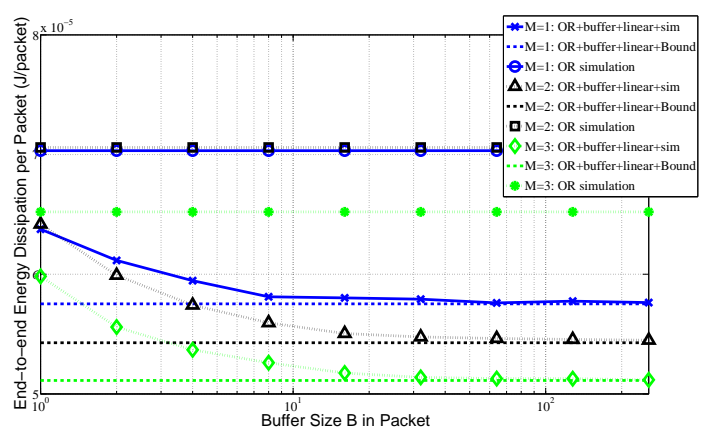

Fig. 5. Average normalized end-to-end energy dissipation (J/packet) as defined in (12) when the pathloss factor is $\alpha=2$ and $M=1,2,3$. The theoretical curve was evaluated from (10).

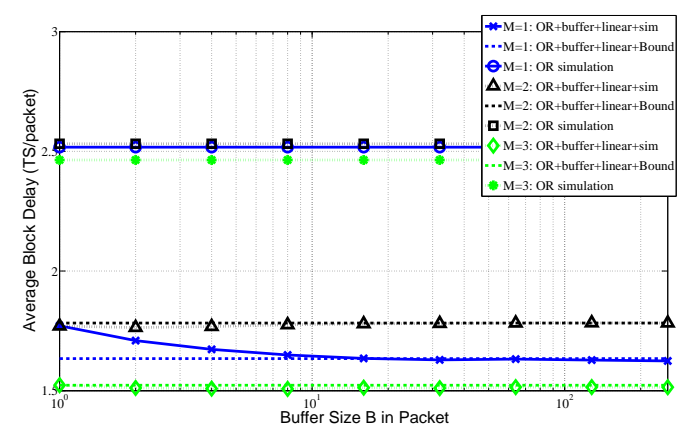

Fig. 6. Block delay (TS/packet) when the pathloss factor is $\alpha=2$ and $M=1,2,3$. The theoretical curve was evaluated from (14).

Fig. 6 was recorded for the same scenario, as Fig. 5. The average block delay is reduced (which means that the throughput is increased) upon adopting buffer-aided transmissions. The main reason is the lower OP of the new scheme, as shown in Fig. 4, where the OP degrades the throughput most dramatically in the context of a fixedrate system.

\section{CONCLUSions}

In this paper, a new buffer-aided OR scheme relying on a novel linear multi-dimensional TAPS partitioning method was proposed, which combines the advantages of buffer-aided transmissions with the benefits of OR, while satisfying the constraint that the number of input packets equals that of the output packets at each RN's buffer. The proposed scheme was shown to outperform the OR scheme both in terms of the normalized end-to-end energy dissipation and the outage probability. Additionally, the simulation based performance of the proposed scheme approaches the theoretical bounds as the RN's buffer size increases.

\section{REFERENCES}

[1] S. Biswas and R. Morris, "ExOR: Opportunistic multi-hop routing for wireless networks," in ACM Special Interest Group on Data Communication (SIGCOMM), Philadelphia, PA, Aug. 2005, pp. 133-144.

[2] C. Dong, L.-L. Yang, and L. Hanzo, "Performance analysis of multihop diversity aided multi-hop links," IEEE Transactions on Vehicular Technology, vol. 61, no. 6, pp. 2504-2516, 2012.

[3] A. Muller and H.-C. Yang, "Dual-hop adaptive packet transmission systems with regenerative relaying," IEEE Transactions on Wireless Communications, vol. 9, no. 1, pp. 234 -244, Jan. 2010.

[4] F. A. Onat, A. Adinoyi, Y. Fan, H. Yanikomeroglu, J. S. Thompson, and I. D. Marsland, "Threshold selection for SNR-based selective digital relaying in cooperative wireless networks," IEEE Transactions on Wireless Communications, vol. 7, no. 11, pp. 4226 -4237, Nov. 2008.

[5] B. Xia, Y. Fan, J. S. Thompson, and H. V. Poor, "Buffering in a threenode relay network," IEEE Transactions on Wireless Communications, vol. 7, no. 11, pp. $4492-4496$, Nov. 2008.

[6] H. Liu, B. Zhang, H. Mouftah, X. Shen, and J. Ma, "Opportunistic routing for wireless Ad Hoc and sensor networks: Present and future directions," IEEE Communications Magazine, vol. 47, no. 12, pp. 103109, 2009.

[7] K. Zeng, Z. Yang, and W. Lou, "Location-aided opportunistic forwarding in multirate and multihop wireless networks," IEEE Transactions on Vehicular Technology, vol. 58, no. 6, pp. 3032-3040, July 2009.

[8] J. Zuo, C. Dong, H. V. Nguyen, S. X. Ng, L.-1. Yang, and L. Hanzo, "Cross-layer aided energy-efficient opportunistic routing in ad hoc networks," IEEE Transactions on Communications, vol. PP, no. 99, pp. 1-14, 2014.

[9] L.-L. Yang, C. Dong, and L. Hanzo, "Multihop diversity-a precious source of fading mitigation in multihop wireless networks," in IEEE Global Telecommunications Conference (Globecom'11), Houston, TX, USA, Dec. 2011, pp. 1-5.

[10] C. Dong, L.-L. Yang, and L. Hanzo, "Multihop diversity for fading mitigation in multihop wireless networks," in IEEE Vehicular Technology Conference (VTC'11-Fall), San Francisco, CA, USA, Sept. 2011, pp. 15.

[11] A. Ikhlef, D. S. Michalopoulos, and R. Schober, "Max-max relay selection for relays with buffers," IEEE Transactions on Wireless Communications, vol. 11, no. 3, pp. $1124-1135$, March 2012.

[12] A. Ikhlef, J. Kim, and R. Schober, "Mimicking full-duplex relaying using half-duplex relays with buffers," IEEE Transactions on Vehicular Technology, vol. 61, no. 7, pp. 3025-3037, Sep. 2012.

[13] N. Zlatanov and R. Schober, "Buffer-aided relaying with adaptive link selection-fixed and mixed rate transmission," IEEE Transactions on Information Theory, vol. 59, no. 5, pp. 2816-2840, 2013.

[14] C. Dong, L.-L. Yang, J. Zuo, S. X. Ng, and L. Hanzo, "Minimum average end-to-end packet energy dissipation of a buffer-aided three-node network relying on opportunistic routing," Submitted to IEEE Transactions on Communications. [Online]. Available: http://eprints.soton.ac.uk/350501/

[15] N. C. Beaulieu and J. Hu, "A closed-form expression for the outage probability of decode-and-forward relaying in dissimilar Rayleigh fading channels," IEEE Communications Letters, vol. 10, no. 12, pp. 813-815, Dec. 2006.

[16] C. Dong, J. Zuo, L.-L. Yang, S. Ng, and L. Hanzo, "Energy-efficient buffer-aided relaying relying on non-linear channel probability space division," in IEEE Wireless Communications and Networking Conference (WCNC'14), Istanbul, Turkey, Apr. 2014, pp. 1-5.

[17] I. Gradshteyn and I. Ryzhik, Table of Integrals, Series, and Products, Seventh Edition. Elsevier Pte Ltd., 2007. 